\title{
Erratum: Stopping power of dense plasmas: The collisional method and limitations of the dielectric formalism [Phys. Rev. E 97, 023202 (2018)]
}

\author{
C. F. Clauser $\odot$ and N. R. Arista
}

(Q) (Received 15 July 2020; published 28 July 2020)

DOI: 10.1103/PhysRevE.102.019903

We wish to correct a misprint in Eq. (B7) of our paper. The correct expression should be the following:

$$
S=\frac{2 \pi m n}{\left(2 \pi v_{s}^{2}\right)^{3 / 2}} \int_{0}^{\infty} d v_{r} v_{r}^{4} \sigma_{\mathrm{tr}}\left(v_{r}\right) \exp \left(-\frac{v^{2}+v_{r}^{2}}{2 v_{s}^{2}}\right) I\left(\frac{v v_{r}}{v_{s}^{2}}\right)
$$

(the only change is the minus sign in the exponential term). This typographical error was also replicated in Eq. (14).

Moreover, we also want to note that in avoiding divergences when calculating the $S$ integral, the term $e^{\beta}$ in the expression of $I(\beta)$, Eq. (B6), should be extracted and combined with the

$$
\exp \left(-\frac{v^{2}+v_{r}^{2}}{2 v_{s}^{2}}\right)
$$

so that the more practical way to calculate the stopping power is

$$
S=\frac{2 \pi m n}{\left(2 \pi v_{s}^{2}\right)^{3 / 2}} \int_{0}^{\infty} d v_{r} v_{r}^{4} \sigma_{\mathrm{tr}}\left(v_{r}\right) \exp \left(-\frac{\left(v-v_{r}\right)^{2}}{2 v_{s}^{2}}\right) J\left(\frac{v v_{r}}{v_{s}^{2}}\right),
$$

where

$$
J(\beta)=\frac{1}{\beta^{2}}\left[(1+\beta) e^{-2 \beta}-(1-\beta)\right] .
$$

Nevertheless, the misprint did not affect computations or conclusions throughout the paper. 